\title{
Exact Moments of order Statistics from The exponentiated Lomax distribution
}

\author{
Nasr Ibrahim Rashwan \\ Faculty of Commerce - Tanta University, Egypt \\ nasrrashwan@yahoo.com
}

\section{Abstract}

In this paper, order statistics from the exponentiated Lomax distribution (ELD) are obtained. Exact form for the single, product and Triple moment of order statistics from ELD are derived. Measures of skewness and kurtosis of the probability density function of the $\mathrm{r}^{\text {th }}$ order statistic are presented. Some recurrence relations for the single and product moments of order statistics from ELD are established. Also, the percentage points of single order statistics from ELD are computed.

\section{Keywords}

Exponentiated Lomax distribution; order statistics; moments of order statistics; recurrence Relations,

percentage points

\section{Introduction}

Order statistics have been used in many applications, including estimation and detection of extreme values, quality control, goodness of fit, robust statistical, analysis of censored sample, etc (more details see Balakirshnan and Choen 1991, Balakirshnan and Chan 1998, David and Nagaraja 2003 and Taher et al 2015).

The moments of order statistics have some important applications in inferential methods. Several authors have studied the probability density function and the moments of order statistics, in addition to the derivation of some recurrence relations of these moments arising from many specific continuous distributions such as pareto, exponential, gamma, logistic, half logistic, Burr Type X, exponentiated Log-logestic and Poisson-Lomax. (more details, see Malik 1966, Joshi and Balakirshnan 1982, Balakirshnan et al 1988, Ragab 1998, Sultan 2007, Khan et al 2008, Shawky and Bakoban 2009 Athar and Nayabuddin 2014, and Al-Zahrani et al 2015).

Abdul-Moniem and Abdel-Hameed (2012) generalized the Lomax distribution by powering a positive real number ( $\alpha$ ) to the cumulative distribution function (cdf). This new family of distributions called exponentiated Lomax distribution (ELD).

A random variable $X$ is said to have on exponentiated Lomax distribution with parameters $\alpha, \lambda$ and $\theta>0$, write $X \sim E$ El $(\alpha, \lambda$, $\theta$ ), if its probability density function (pdf) is given by

$f(x ; \alpha, \lambda, \theta)=\alpha \lambda \theta\left[1-(1+\lambda x)^{-\theta}\right]^{\alpha-1}[1+\lambda x]^{-(\theta+1)}, x>0, \alpha, \lambda$ and $\theta>0$

and the cumulative distribution function (cdf) of $X$ is given by

$F(x ; \alpha, \lambda, \theta)=\left[1-(1+\lambda x)^{-\theta}\right]^{\alpha}, x>0, \alpha, \lambda$ and $\theta>0$

Where $\alpha$ and $\theta$ are the shape parameters and $\lambda$ is the scale parameter. The survival function $S(x)$, hazard rate function $h(x)$, reversed hazard rate function $r(x)$ and the cumulative hazard rate function $H(x)$ of ELD are given by

$$
\begin{aligned}
& \mathrm{S}(\mathrm{x} ; \alpha, \lambda, \theta)=1-\mathrm{F}(\mathrm{x} ; \alpha, \lambda, \theta)=1-\left[1-(1+\lambda \mathrm{x})^{-\theta}\right]^{\alpha}, \\
& \mathrm{h}(\mathrm{x} ; \alpha, \lambda, \theta)=\frac{\mathrm{f}(\mathrm{x} ; \alpha, \lambda, \theta)}{\mathrm{S}(\mathrm{x} ; \alpha, \lambda, \theta)}=\frac{\alpha \lambda \theta\left[1-(1+\lambda \mathrm{x})^{-\theta}\right]^{\alpha-1}[1+\lambda \mathrm{x}]}{1-\left[1-(1+\lambda \mathrm{x})^{-\theta}\right]^{\alpha}} \\
& \mathrm{r}(\mathrm{x} ; \alpha, \lambda, \theta)=\frac{\mathrm{f}(\mathrm{x} ; \alpha, \lambda, \theta)}{\mathrm{F}(\mathrm{x} ; \alpha, \lambda, \theta)}=\frac{\alpha \lambda \theta[1+\lambda \mathrm{x}]^{-(\theta+1)}}{1-(1+\lambda \mathrm{x})^{-\theta}} \\
& \text { and }
\end{aligned}
$$

$$
H(x ; \alpha, \lambda, \theta)=-\ln S(x)=-\ln \left(1-\left[1-(1+\lambda x)^{-\theta}\right]^{\alpha}\right)
$$

Note that, when $\alpha=1$, the pdf of the ELD reduces to Lomax distribution, $\lambda=1$, the pdf of the ELD reduces, to exponentiated pareto distribution and $\alpha=\lambda=1$ reduce to standard pareto distribution.

In this paper, we obtain exact form expressions for the pdf of order statistics for ELD in section 2 . In section 3 , we derive exact expressions for the single, product and triple moments for order statistics from ELD and compute the measures of skewness and kurtosis of the pdf of the $r^{\text {th }}$ order statistics. We establish some recurrence relations for the single and 
product moments for order statistics from ELD in section 4. Section 5, gives the percentage points of the $r^{\text {th }}$ order statistics. Finally, some conclusions are addressed in section 6.

\section{Distribution of order statistics}

Let $x_{1}, x_{2}, \ldots, x_{n}$ be a random sample of size $n$ from ELD with pdf and cdf as in (1) and (2) respectively, and let $x_{1: n}, x_{2: n}, \ldots$, $x_{n: n}$ denote the corresponding order statistics. Then the pdf of $x_{r: n}, 1 \leq r \leq n$, is given by (Arnold et al 1992 and David and Nagaraja 2003)

$f_{r: n}(x)=C_{r: n}[F(x)]^{r-1}[1-F(x)]^{n-r} f(x) \quad x>0$

Where $C_{r: n}=\frac{n !}{(r-1) !(n-r) !}=[B(r, n-r+1)]^{-1}, \quad$ with $B(a, b)$ being the complete beta

function and $f(x)=f(x ; \alpha, \lambda, \theta)$ and $F(x)=F(x ; \alpha, \lambda, \theta)$ are pdf and cdf given in (1) and (2).

Theorem 2.1. Let $f(x)$ and $F(x)$ be the pdf and cdf of ELD for a random variable $X$. Then the pdf of the $r^{\text {th }}$ order statistic say $f_{r: n}$ (x) is given by

$\mathrm{f}_{\mathrm{r}: \mathrm{n}}(\mathrm{x})=\sum_{\mathrm{i}=0}^{\mathrm{n}-\mathrm{r}} \mathrm{d}_{\mathrm{i}}(\mathrm{n}, \mathrm{r}) \mathrm{f}(\mathrm{x} ; \alpha(\mathrm{i}+\mathrm{r}), \lambda, \theta)$

Proof: by using binomial expansion, the pdf in (7) can be written as

$\mathrm{f}_{\mathrm{r}: \mathrm{n}}(\mathrm{x})=\mathrm{C}_{\mathrm{r}: \mathrm{n}} \sum_{\mathrm{i}=0}^{\mathrm{n}-\mathrm{r}}(-1)^{\mathrm{i}}\left(\begin{array}{c}\mathrm{n}-\mathrm{r} \\ \mathrm{i}\end{array}\right)[\mathrm{F}(\mathrm{x})]^{\mathrm{i}+\mathrm{r}-1} \mathrm{f}(\mathrm{x})$

$\mathrm{f}_{\mathrm{r}: \mathrm{n}}(\mathrm{x})=\mathrm{C}_{\mathrm{r}: \mathrm{n}} \sum_{\mathrm{i}=0}^{\mathrm{n}-\mathrm{r}}(-1)^{\mathrm{i}}\left(\begin{array}{c}\mathrm{n}-\mathrm{r} \\ \mathrm{i}\end{array}\right) \alpha \theta \lambda\left[1-(1+\lambda \mathrm{x})^{-\theta}\right]^{\alpha(\mathrm{i}+\mathrm{r})-1}[1+\lambda \mathrm{x}]^{-(\theta+1)}$

Let $d_{i}(n, r)=\frac{\left(\begin{array}{c}n-r \\ i\end{array}\right)\left(\begin{array}{c}n-1 \\ r-1\end{array}\right)}{(i+r)} \quad, \quad 1 \leq r \leq n$

Then $\mathrm{f}_{\mathrm{r}: \mathrm{n}}(\mathrm{x})=\sum_{\mathrm{i}=0}^{\mathrm{n}-\mathrm{r}} \mathrm{d}_{\mathrm{i}}(\mathrm{n}, \mathrm{r}) \mathrm{f}(\mathrm{x} ; \alpha(\mathrm{i}+\mathrm{r}), \lambda, \theta) \quad, \quad \mathrm{x}>0$

Note that, $d_{i}(n, r),(i=0,1,2, \ldots, n-r)$, are coefficients not dependent on $\alpha, \lambda$ and $\theta$. This observation means that $f_{r: n}(x)$ is a weighted average of exponentiated lomax densities. As special cases of (8), the pdf of the smallest $(r=1)$ and the largest $(r=n)$ order statistics can be easily obtained as

$$
f_{1: n}(x)=\sum_{i=0}^{n-1} \frac{n(-1)^{i}\left(\begin{array}{c}
n-1 \\
i
\end{array}\right)}{(i+1)} f(x ; \alpha(i+1), \lambda, \theta) \quad, \quad x>0
$$

and

$$
\mathrm{f}_{\mathrm{n}: \mathrm{n}}(\mathrm{x})=\mathrm{f}(\mathrm{x} ; \text { an, } \lambda, \theta) \quad \mathrm{x}>0
$$

The joint pdf of any two order statistics $\mathrm{x}=\mathrm{x}_{\mathrm{r}: \mathrm{n}}$ and $\mathrm{y}=\mathrm{x}_{\mathrm{s}: \mathrm{n}}$ for $1 \leq \mathrm{r}<\mathrm{s} \leq \mathrm{n}$ is given by

$\mathrm{f}_{\mathrm{r}, \mathrm{s}: \mathrm{n}}(\mathrm{x}, \mathrm{y})=\mathrm{C}_{\mathrm{r}, \mathrm{s}: \mathrm{n}}[\mathrm{F}(\mathrm{x})]^{\mathrm{r}-1}[\mathrm{~F}(\mathrm{y})-\mathrm{F}(\mathrm{x})]^{\mathrm{s}-\mathrm{r}-1}[1-\mathrm{F}(\mathrm{y})]^{\mathrm{n}-\mathrm{s}} \mathrm{f}(\mathrm{x}) \mathrm{f}(\mathrm{y}), 0<\mathrm{x}<\mathrm{y}<\infty$ 
Where $C_{r, s: n}=\frac{n !}{(r-1) !(s-r-1) !(n-s) !}$

Theorem 2.2. Let $X_{r: n}$ and $X_{s: n}$ for $1 \leq r<s \leq n$ be the $r^{\text {th }}$ and $s^{\text {th }}$ order statistics for the ELD. Then the joint pdf of $X_{\mathrm{r}: n}$ and $X_{\mathrm{s}: \mathrm{n}}$ is given by

$$
\begin{aligned}
\mathrm{f}_{\mathrm{r}, \mathrm{s}: n}(\mathrm{x}, \mathrm{y})=\mathrm{C}_{\mathrm{r}, \mathrm{s}: n} & \sum_{\mathrm{i}=0}^{\mathrm{s}-\mathrm{r}-1} \sum_{\mathrm{j}=0}^{\mathrm{n}-\mathrm{s}} \frac{(-1)^{\mathrm{i}+\mathrm{j}}\left(\begin{array}{c}
\mathrm{s}-\mathrm{r}-1 \\
\mathrm{i}
\end{array}\right)\left(\begin{array}{c}
\mathrm{n}-\mathrm{s} \\
\mathrm{j}
\end{array}\right)}{(\mathrm{i}+\mathrm{r})(\mathrm{s}-\mathrm{r}-\mathrm{i}+\mathrm{j})} \mathrm{f}(\mathrm{x} ; \alpha(\mathrm{i}+\mathrm{r}), \lambda, \theta) \\
& \times \mathrm{f}(\mathrm{y} ; \alpha(\mathrm{s}-\mathrm{r}-\mathrm{i}+\mathrm{j}), \lambda, \theta) \quad, \quad 0<\mathrm{x}<\mathrm{y}<\infty
\end{aligned}
$$

proof: By using binomial expansion, the pdf in (9) can be written as

$$
\mathrm{f}_{\mathrm{r}, \mathrm{s}: \mathrm{n}}(\mathrm{x}, \mathrm{y})=\mathrm{C}_{\mathrm{r}, \mathrm{s}: \mathrm{n}} \sum_{\mathrm{i}=0}^{\mathrm{s}-\mathrm{r}-1} \sum_{\mathrm{j}=0}^{\mathrm{n}-\mathrm{s}}(-1)^{\mathrm{i}+\mathrm{j}}\left(\begin{array}{c}
\mathrm{s}-\mathrm{r}-1 \\
\mathrm{i}
\end{array}\right)\left(\begin{array}{c}
\mathrm{n}-\mathrm{s} \\
\mathrm{j}
\end{array}\right)[\mathrm{F}(\mathrm{y})]^{\mathrm{s}-\mathrm{r}-1-\mathrm{i}+\mathrm{j}}[\mathrm{F}(\mathrm{x})]^{\mathrm{i}+\mathrm{r}-1} \mathrm{f}(\mathrm{x}) \mathrm{f}(\mathrm{y})
$$

By substituting (1) and (2) in the previous equation, then

$$
\begin{aligned}
f_{r, s: n}(x, y)= & C_{r, s: n} \alpha^{2} \theta^{2} \lambda^{2} \sum_{i=0}^{s-r-1} \sum_{j=0}^{n-s}(-1)^{i+j}\left(\begin{array}{c}
s-r-1 \\
i
\end{array}\right)\left(\begin{array}{c}
n-s \\
j
\end{array}\right)\left[1-(1+\lambda x)^{-\theta}\right]^{\alpha(i+r)-1} \\
\times & {[1+\lambda x]^{-(\theta+1)}\left[1-(1+\lambda x)^{-\theta}\right]^{\alpha(s-r-i+j)-1}[1+\lambda y]^{-(\theta+1)}, 0<x<y<\infty } \\
= & C_{r, s: n} \sum_{i=0}^{s-r-1} \sum_{j=0}^{n-s}(-1)^{i+j} \frac{\left(\begin{array}{c}
s-r-1 \\
i
\end{array}\right)\left(\begin{array}{c}
n-s \\
j
\end{array}\right)}{(i+r)(s-r-i+j)} f(x ; \alpha(i+r), \lambda, \theta) \\
& \times f(y ; \alpha(s-r-i+j), \lambda, \theta), 0<x<y<\infty
\end{aligned}
$$

Using the same method that used to prove theories (2.1) and (2.2), we can get the joint pdf of three order statistics as follows:

Theorem 2.3. Let $X_{r: n}, X_{s: n}$ and $X_{t: n}$ for $1 \leq r<s<t \leq n$ be the $r^{\text {th }}, s^{\text {th }}$ and $t^{\text {th }}$ order statistics from the ELD. Then the joint pdf of $\mathrm{X}_{\mathrm{r}: \mathrm{n}}, \mathrm{X}_{\mathrm{s}: \mathrm{n}}$ and $\mathrm{X}_{\mathrm{t}: \mathrm{n}}$ is given by

$$
\begin{aligned}
\mathrm{f}_{\mathrm{r}, \mathrm{s}, \mathrm{t}: \mathrm{n}}(\mathrm{x}, \mathrm{y}, \mathrm{z}) & =\mathrm{C}_{\mathrm{r}, \mathrm{s}, \mathrm{t}: \mathrm{n}}[\mathrm{F}(\mathrm{x})]^{\mathrm{r}-1}[\mathrm{~F}(\mathrm{y})-\mathrm{F}(\mathrm{x})]^{\mathrm{s}-\mathrm{r}-1}[\mathrm{~F}(\mathrm{z})-\mathrm{F}(\mathrm{y})]^{\mathrm{t}-\mathrm{s}-1}[1-\mathrm{F}(\mathrm{z})]^{\mathrm{n}-\mathrm{t}} \\
& \times \mathrm{f}(\mathrm{x}) \mathrm{f}(\mathrm{y}) \mathrm{f}(\mathrm{z}), 0<\mathrm{x}<\mathrm{y}<\mathrm{z}<\infty
\end{aligned}
$$

Where $\mathrm{C}_{\mathrm{r}, \mathrm{s}, \mathrm{t}: \mathrm{n}}=\frac{\mathrm{n} !}{(\mathrm{r}-1) !(\mathrm{s}-\mathrm{r}-1) !(\mathrm{t}-\mathrm{s}-1) !(\mathrm{n}-\mathrm{t}) !}$

By substituting (1) and (2) in (11), then

$$
\begin{aligned}
& \mathrm{f}_{r, s, t: n}(x, y, z)=C_{r, s, t: n} \alpha^{3} \theta^{3} \lambda^{3} \sum_{i=0}^{s-r-1} \sum_{j=0}^{t-s-1} \sum_{m=0}^{n-t}(-1)^{i+j+m}\left(\begin{array}{c}
s-r-1 \\
i
\end{array}\right)\left(\begin{array}{c}
t-s-1 \\
j
\end{array}\right)\left(\begin{array}{c}
n-t \\
m
\end{array}\right) \\
& \times(1+\lambda x)^{-(\theta+1)}\left[1-(1+\lambda x)^{-\theta}\right]^{\alpha(i+r)-1}(1+\lambda y)^{-(\theta+1)}\left[1-(1+\lambda y)^{-\theta}\right]^{\alpha(s-r-i+j)-1} \\
& \times(1+\lambda z)^{-(\theta+1)}\left[1-(1+\lambda z)^{-\theta}\right]^{\alpha(t-s-j+m)-1}, 0<x<y<z<\infty
\end{aligned}
$$




$$
\begin{aligned}
\mathrm{f}_{\mathrm{r}, \mathrm{s}: \mathrm{t}: \mathrm{n}}(\mathrm{x}, \mathrm{y}, \mathrm{z})=\mathrm{C}_{\mathrm{r}, \mathrm{s}, \mathrm{t}: \mathrm{n}} \sum_{\mathrm{i}=0}^{\mathrm{s}-\mathrm{r}-1} \sum_{\mathrm{j}=0}^{\mathrm{t}-\mathrm{s}-1} \sum_{\mathrm{m}=0}^{\mathrm{n}-\mathrm{t}}(-1) \frac{\left(\begin{array}{c}
\mathrm{s}-\mathrm{r}-1 \\
\mathrm{i}
\end{array}\right)\left(\begin{array}{c}
\mathrm{t}-\mathrm{s}-1 \\
\mathrm{j}
\end{array}\right)\left(\begin{array}{c}
\mathrm{n}-\mathrm{t} \\
\mathrm{m}
\end{array}\right)}{(\mathrm{i}+\mathrm{r})(\mathrm{s}-\mathrm{r}-\mathrm{i}+\mathrm{j})(\mathrm{t}-\mathrm{s}-\mathrm{j}+\mathrm{m})} \\
\times \mathrm{f}(\mathrm{x} ; \alpha(\mathrm{i}+\mathrm{r}), \lambda, \theta) \mathrm{f}(\mathrm{y} ; \alpha(\mathrm{s}-\mathrm{r}-\mathrm{i}+\mathrm{j}), \lambda, \theta) \mathrm{f}(\mathrm{z} ; \alpha(\mathrm{t}-\mathrm{s}-\mathrm{j}+\mathrm{m}), \lambda, \theta), \\
0<\mathrm{x}<\mathrm{y}<\mathrm{z}<\infty
\end{aligned}
$$

\section{Moments of order statistics}

In this section, we derive a closed form expressions for the single, product and triple moments for order statistics from ELD.

\subsection{Single moments}

Theorem 3.1. Let $\mathrm{x}_{1}, \mathrm{x}_{2}, \ldots, \mathrm{x}_{\mathrm{n}}$ be a random sample of size $\mathrm{n}$ from the ELD and let $\mathrm{x}_{1: \mathrm{n}}, \mathrm{x}_{2: \mathrm{n}}, \ldots, \mathrm{x}_{\mathrm{n}: \mathrm{n}}$ denote the corresponding order statistics. Then the $\mathrm{k}^{\text {th }}$ moment of the $\mathrm{r}^{\text {th }}$ order statistic for $\mathrm{k}=1,2, \ldots$, denoted by $\mu_{\mathrm{r}: \mathrm{n}}^{(\mathrm{k})}, 1 \leq \mathrm{r} \leq \mathrm{n}$ is given by

$\mu_{r: n}^{(k)}=E\left(x_{r: n}^{k}\right)=\sum_{i=0}^{n-r} \sum_{j=0}^{k}(-1)^{k+j}\left(\begin{array}{c}k \\ j\end{array}\right) d_{i}(n, r) \lambda^{-k} \alpha(i+r) B\left[\alpha(i+r), 1-\frac{j}{\theta}\right] \cdot \theta>k$

Proof: we know that

$$
\begin{aligned}
\mu_{r: n}^{(k)} & =\int_{0}^{\infty} x^{k} f_{r: n}(x) d x \\
& =\sum_{i=0}^{n-r} d_{i}(n, r) \alpha(i+r) \theta \lambda \int_{0}^{\infty} x^{k}\left[1-(1+\lambda x)^{-\theta}\right]^{\alpha(i+r)-1}[1+\lambda x]^{-(\theta+1)} d x
\end{aligned}
$$

Let $(1+\lambda x)^{-\theta}=y ، \theta \lambda(1+\lambda x)^{-(\theta+1)} \mathrm{dx}=-\mathrm{dy} ، \quad \mathrm{x}=\left(\mathrm{y}^{-1 / \theta}-1\right) / \lambda(1<\mathrm{y}<0$, then

$$
\mu_{r: n}^{(k)}=\sum_{i=0}^{n-r} d_{i}(n, r) \lambda^{-k} \alpha(i+r) \int_{0}^{1}\left(y^{-1 / \theta}-1\right)^{k}(1-y)^{\alpha(i+r)-1} d y
$$

By using binomial expansion, then

$$
\begin{aligned}
\mu_{r: n}^{(k)} & =\sum_{i=0}^{n-r} d_{i}(n, r) \lambda^{-k} \alpha(i+r) \sum_{j=0}^{k}(-1)^{k+j}\left(\begin{array}{c}
k \\
j
\end{array}\right) \int_{0}^{1} y^{-j / \theta}(1-y)^{\alpha(i+r)-1} d y \\
& =\sum_{i=0}^{n-r} \sum_{j=0}^{k}(-1)^{k+j}\left(\begin{array}{c}
k \\
j
\end{array}\right) d_{i}(n, r) \lambda^{-k} \alpha(i+r) B[\alpha(i+r), 1-j / \theta], \quad \theta>k
\end{aligned}
$$

Also, we can prove that

$$
\begin{aligned}
\mu_{r: n}^{(k)}= & \sum_{i=0}^{n-r} \sum_{j=0}^{k} \sum_{m=0}^{\alpha(i+r)-1}(-1)^{i+j+m}\left(\begin{array}{c}
k \\
j
\end{array}\right)\left(\begin{array}{c}
\alpha(i+r)-1 \\
m
\end{array}\right) d_{i}(n, r) \lambda^{-k} \\
& \times \alpha(i+r)\left[\frac{\theta}{j-k+m \theta+\theta}\right] \text { for } k<j+\theta+m \theta
\end{aligned}
$$

Let $(1+\lambda \mathrm{x})^{-\theta}=\mathrm{y}$ ، $\theta \lambda(1+\lambda \mathrm{x})^{-(\theta+1)} \mathrm{dx}=-\mathrm{dy}, 1<\mathrm{y}<0, \mathrm{x}=\left(\mathrm{y}^{-1 / \theta}-1\right) / \lambda$ ، then 
$\mu_{\mathrm{r}: \mathrm{n}}^{(\mathrm{k})}=\sum_{\mathrm{i}=0}^{\mathrm{n}-\mathrm{r}} \mathrm{d}_{\mathrm{i}}(\mathrm{n}, \mathrm{r}) \lambda^{-\mathrm{k}} \alpha(\mathrm{i}+\mathrm{r}) \int_{0}^{1} \mathrm{y}^{-\mathrm{k} / \theta}\left(1-\mathrm{y}^{-1 / \theta}\right)^{\mathrm{k}}(1-\mathrm{y})^{\alpha(\mathrm{i}+\mathrm{r})-1} \mathrm{dy}$

By using binomial expansion, then

$$
\begin{aligned}
& \mu_{r: n}^{(k)}=\sum_{i=0}^{n-r} \sum_{j=0}^{k} \sum_{m=0}^{\alpha(i+r)-1}(-1)^{j+m}\left(\begin{array}{c}
k \\
j
\end{array}\right)\left(\begin{array}{c}
\alpha(i+r)-1 \\
m
\end{array}\right) d_{i}(n, r) \lambda^{-k} \alpha(i+r) \int_{0}^{1} y^{\left(\frac{j-k+m \theta}{\theta}\right)} d y \\
& =\sum_{i=0}^{n-r} \sum_{j=0}^{k} \sum_{m=0}^{\alpha(i+r)-1}(-1)^{j+m}\left(\begin{array}{c}
k \\
j
\end{array}\right)\left(\begin{array}{c}
\alpha(i+r)-1 \\
m
\end{array}\right) d_{i}(n, r) \lambda^{-k} \alpha(i+r)\left[\frac{\theta}{j-k+m \theta+\theta}\right]
\end{aligned}
$$$$
\mathrm{k}<\mathrm{j}+\theta+\mathrm{m} \theta
$$

for $\mathrm{n}=\mathrm{r}$, formula in (13) reduces to

$\mu_{\mathrm{r}: \mathrm{r}}^{(\mathrm{k})}=\mathrm{r} \sum_{\mathrm{j}=0}^{\mathrm{k}}(-1)^{\mathrm{k}+\mathrm{j}}\left(\begin{array}{c}\mathrm{k} \\ \mathrm{j}\end{array}\right) \lambda^{-\mathrm{k}}$ ar $\mathrm{B}[\alpha \mathrm{r}, 1-\mathrm{j} / \theta]$

and $\mathrm{r}=\mathrm{n}$, formula in (13) reduces to

$$
\mu_{n: n}^{(k)}=\sum_{j=0}^{k}(-1)^{k+j}\left(\begin{array}{l}
k \\
j
\end{array}\right) \lambda^{-k} \text { an } B[\alpha n, 1-j / \theta]
$$

Moreover, the measures of skewness (sk) and kurtosis $(\mathrm{ku})$ of the distribution of the $\mathrm{r}^{\text {th }}$ order statistic can be computed from the following equations

$$
\mathrm{sk}=\frac{\mu_{\mathrm{r}: \mathrm{n}}^{(3)}-3 \mu_{\mathrm{r}: \mathrm{n}} \mu_{\mathrm{r}: \mathrm{n}}^{(2)}+2 \mu_{\mathrm{r}: \mathrm{n}}^{3}}{\left[\mu_{\mathrm{r}: \mathrm{n}}^{(2)}-\mu_{\mathrm{r}: \mathrm{n}}^{2}\right]^{3 / 2}}
$$

and

$$
\mathrm{ku}=\frac{\mu_{\mathrm{r}: \mathrm{n}}^{(4)}-4 \mu_{\mathrm{r}: \mathrm{n}} \mu_{\mathrm{r}: \mathrm{n}}^{(3)}+6 \mu_{\mathrm{r}: \mathrm{n}}^{2} \mu_{\mathrm{r}: \mathrm{n}}^{(2)}-3 \mu_{\mathrm{r}: \mathrm{n}}^{4}}{\left[\mu_{\mathrm{r}: \mathrm{n}}^{(2)}-\mu_{\mathrm{r}: \mathrm{n}}^{2}\right]^{2}}
$$

Note that the variance of $X_{r: n}=\mu_{r: n}^{(2)}-\stackrel{2}{\mu}$

The following table (1) shows the values of sk and ku of $X_{\mathrm{r}: \mathrm{n}}$ for different values of $\theta=5,7, \lambda=2,4$ and $\alpha=2,3$ when $\mathrm{n}=2,3,5$ and $1 \leq \mathrm{r} \leq \mathrm{n}$. 
Table (1): Values of sk and ku for different values of $\lambda, \alpha, \theta$

\begin{tabular}{|c|c|c|c|c|c|}
\hline \multicolumn{2}{|c|}{$\theta=5$} & \multirow{2}{*}{$\begin{array}{c}\lambda=2 \\
\text { sk }\end{array}$} & \multirow{2}{*}{$\begin{array}{c}\alpha=2 \\
k u\end{array}$} & \multirow{2}{*}{$\begin{array}{c}\lambda=4 \\
\text { sk }\end{array}$} & \multirow{2}{*}{$\frac{\alpha=3}{k u}$} \\
\hline $\mathbf{n}$ & $\mathbf{r}$ & & & & \\
\hline \multirow[t]{2}{*}{2} & 1 & 2.44 & 14.03 & 2.26 & 8.84 \\
\hline & 2 & 3.71 & 53.126 & 3.65 & 51.88 \\
\hline \multirow[t]{3}{*}{3} & 1 & 1.71 & 325.125 & 1.34 & 6.49 \\
\hline & 2 & 2.08 & 11.29 & 1.64 & 8.42 \\
\hline & 3 & 3.66 & 51.88 & 3.68 & $\mathbf{5 0 . 5 7}$ \\
\hline \multirow[t]{5}{*}{5} & 1 & 1.33 & 6.54 & 1.84 & 3.97 \\
\hline & 2 & 0.609 & 6.67 & 1.12 & 3.38 \\
\hline & 3 & 0.735 & 4.27 & 1.37 & 3.63 \\
\hline & 4 & 1.59 & 7.54 & 1.68 & 8.08 \\
\hline & 5 & 3.72 & 51.11 & 3.62 & 49.6 \\
\hline \multicolumn{2}{|c|}{$\theta=7$} & $\lambda=2$ & $\alpha=2$ & $\lambda=4$ & $\alpha=3$ \\
\hline n & $\mathbf{r}$ & Sk & $\mathbf{k u}$ & sk & ku \\
\hline \multirow[t]{2}{*}{2} & 1 & 1.462 & 15.96 & 1.82 & 14.81 \\
\hline & 2 & 2.76 & 18.82 & 2.86 & 18.28 \\
\hline \multirow[t]{3}{*}{3} & 1 & 4.67 & 5.51 & 2.81 & 4.22 \\
\hline & 2 & 1.35 & 12.32 & 2.49 & 14.66 \\
\hline & 3 & 2.65 & 18.28 & 2.33 & 18.47 \\
\hline \multirow[t]{5}{*}{5} & 1 & 0.89 & 4.25 & 5.86 & 9.86 \\
\hline & 2 & 0.87 & 4.35 & 0.56 & 7.34 \\
\hline & 3 & 1.89 & 12.72 & 2.84 & 5.18 \\
\hline & 4 & 1.56 & 7.62 & 1.63 & 8.94 \\
\hline & 5 & 2.77 & 18.23 & 2.50 & 17.78 \\
\hline
\end{tabular}

From table (1), for these selected values, we notice that the distribution of the $\mathrm{r}^{\text {th }}$ order statistic is positively skewed because all values of sk more than zero. Also, the distribution of the $\mathrm{r}^{\text {th }}$ order statistic is leptokurtic (higher and sharper peaked than the normal distribution) because all values of ku more than 3 .

\subsection{Product moments}

Theorem 3.2. for the exponentiated lomax distribution as given in (10) and $\alpha, \lambda$ and $\theta>0,1 \leq \mathrm{r}<\mathrm{s} \leq \mathrm{n}$, we have that

$$
\begin{aligned}
\mu_{r, s: n}^{\left(k_{1}, k_{2}\right)} & =C_{r, s: n} \alpha^{2} \theta^{2} \sum_{i=0}^{s-r-1} \sum_{j=0}^{n-s} \sum_{L=0}^{k_{2}} \sum_{m=0}^{\alpha(s-r-i+j)-1} \sum_{p=0}^{k_{1}} \sum_{q=0}^{\alpha(i+r)-1}(-1)^{i+j+L+m+p+q} \\
& \times \lambda^{-\left(\mathrm{k}_{1}+\mathrm{k}_{2}\right)}\left(\begin{array}{c}
\mathrm{s}-\mathrm{r}-1 \\
\mathrm{i}
\end{array}\right)\left(\begin{array}{c}
\mathrm{n}-\mathrm{s} \\
\mathrm{j}
\end{array}\right)\left(\begin{array}{c}
\mathrm{k}_{2} \\
\mathrm{~L}
\end{array}\right)\left(\begin{array}{c}
\alpha(\mathrm{s}-\mathrm{r}-\mathrm{i}+\mathrm{j})-1 \\
\mathrm{~m}
\end{array}\right)\left(\begin{array}{c}
\mathrm{k}_{1} \\
\mathrm{p}
\end{array}\right)\left(\begin{array}{c}
\alpha(\mathrm{i}+\mathrm{r})-1 \\
\mathrm{q}
\end{array}\right) \\
& \times\left[\frac{1}{\left(\mathrm{~L}-\mathrm{k}_{2}+\mathrm{m} \theta+\theta\right)\left(\mathrm{L}+\mathrm{p}-\mathrm{k}_{1}-\mathrm{k}_{2}+\mathrm{m} \theta+\mathrm{q} \theta+2 \theta\right)}\right]
\end{aligned}
$$


Proof: we know that

$$
\begin{aligned}
\mu_{\mathrm{r}, \mathrm{s}: \mathrm{n}}^{\left(\mathrm{k}_{1}, \mathrm{k}_{2}\right)}= & \alpha^{2} \theta^{2} \lambda^{2} \mathrm{C}_{\mathrm{r}, \mathrm{s}: \mathrm{n}} \sum_{\mathrm{i}=0}^{\mathrm{s}-\mathrm{r}-1} \sum_{\mathrm{j}=0}^{\mathrm{n}-\mathrm{s}}(-1)^{\mathrm{i}+\mathrm{j}}\left(\begin{array}{c}
\mathrm{s}-\mathrm{r}-1 \\
\mathrm{i}
\end{array}\right)\left(\begin{array}{c}
\mathrm{n}-\mathrm{s} \\
\mathrm{j}
\end{array}\right) \int_{0}^{\infty} \mathrm{x}^{\mathrm{k}_{1}}\left[1-(1+\lambda \mathrm{x})^{-\theta}\right]^{\alpha(\mathrm{i}+\mathrm{r})-1} \\
& \times[1+\lambda \mathrm{x}]^{-(\theta+1)} \mathrm{I}(\mathrm{x}) \mathrm{dx}
\end{aligned}
$$

Where $I(x)=\int_{x}^{\infty} y^{k_{2}}(1+\lambda y)^{-(\theta+1)}\left[1-(1+\lambda x)^{-\theta}\right]^{\alpha(s-r-i+j)-1} d y$

Put $(1+\lambda \mathrm{y})^{-\theta}=\mathrm{z} \cdot \lambda \theta(1+\lambda \mathrm{y})^{-(\theta+1)} \mathrm{dy}=-\mathrm{dz} \cdot(1+\lambda \mathrm{x})^{-\theta}<\mathrm{y}<0, \quad \mathrm{y}=\left(\mathrm{z}^{-1 / \theta}-1\right) / \lambda$,

Then $I(x)=\frac{\lambda^{-k_{2}}}{\lambda \theta} \int_{0}^{(1+\lambda x)-\theta} z^{-\frac{k_{2}}{\theta}}\left(1-z^{1 / \theta}\right)^{k_{2}}(1-z)^{\alpha(s-r-i+j)-1} d z$

$I(x)=\lambda^{-k_{2}-1} \sum_{L=0}^{k_{2}} \sum_{m=0}^{\alpha(s-r-i+j)-1}(-1)^{L+m}\left(\begin{array}{c}k_{2} \\ L\end{array}\right)\left(\begin{array}{c}\alpha(s-r-i+j)-1 \\ m\end{array}\right)\left[\frac{1}{L-k_{2}+m \theta+\theta}\right]$

$$
\times\left[(1+\lambda x)^{-\theta}\right]\left(\frac{\mathrm{L}-\mathrm{k}_{2}+\mathrm{m} \theta+\theta}{\theta}\right)
$$

Substituting I (x) in (17-A), then

$$
\begin{aligned}
& \mu_{\mathrm{r}, \mathrm{s}: \mathrm{n}}^{\left(\mathrm{k}_{1}, \mathrm{k}_{2}\right)}=\alpha^{2} \theta^{2} \lambda \sum_{\mathrm{i}=0}^{\mathrm{s}-\mathrm{r}-1} \sum_{\mathrm{j}=0}^{\mathrm{n}-\mathrm{s}} \sum_{\mathrm{L}=0}^{\mathrm{k}_{2}} \sum_{\mathrm{m}=0}^{\alpha(\mathrm{s}-\mathrm{r}-\mathrm{i}+\mathrm{j})-1}(-1)^{\mathrm{i}+\mathrm{j}+\mathrm{L}+\mathrm{m}} \lambda^{-\mathrm{k}_{2}}\left(\begin{array}{c}
\mathrm{s}-\mathrm{r}-1 \\
\mathrm{i}
\end{array}\right)\left(\begin{array}{c}
\mathrm{n}-\mathrm{s} \\
\mathrm{j}
\end{array}\right)\left(\begin{array}{c}
\mathrm{k}_{2} \\
\mathrm{~L}
\end{array}\right) \\
& \quad \times\left(\begin{array}{c}
\alpha(\mathrm{s}-\mathrm{r}-\mathrm{i}+\mathrm{j})-1 \\
\mathrm{~m}
\end{array}\right) \int_{0}^{\infty} \mathrm{x}^{\mathrm{k}_{1}}\left[1-(1+\lambda \mathrm{x})^{-\theta}\right]^{\alpha(\mathrm{i}+\mathrm{r})-1}(1+\lambda \mathrm{x})^{-(\theta+1)}\left[(1+\lambda \mathrm{x})^{-\theta}\right]^{\left(\frac{\mathrm{L}-\mathrm{k}_{2}+\mathrm{m} \theta+\theta}{\theta}\right)} \mathrm{dx}
\end{aligned}
$$

Put $(1+\lambda \mathrm{y})^{-\theta}=\mathrm{w} \cdot \theta \lambda(1+\lambda \mathrm{x})^{-(\theta+1)} \mathrm{dx}=-\mathrm{dw} \cdot 1<\mathrm{w}<0, \quad \mathrm{x}=\left(\mathrm{w}^{-1 / \theta}-1\right) / \lambda$

By using both integration by substitute and binomial expansion we get (17).

\subsection{Triple moments}

For exponentiated lomax distribution as given in (12) and $\alpha, \lambda$ and $\theta>0$

and $1 \leq \mathrm{r}<\mathrm{s}<\mathrm{t} \leq \mathrm{n}$, we have that

$$
\begin{aligned}
\underset{\mathrm{r}, \mathrm{s}, \mathrm{t}: \mathrm{n}}{\left(\mathrm{k}_{1}, \mathrm{k}_{2}, \mathrm{k}_{3}\right)} & =\mathrm{C}_{\mathrm{r}, \mathrm{s}, \mathrm{t} \text {. }} \alpha^{3} \theta^{3} \lambda^{-\left(\mathrm{k}_{1}+\mathrm{k}_{2}+\mathrm{k}_{3}\right)} \sum_{\mathrm{i}=0}^{\mathrm{s}-\mathrm{r}-1} \sum_{\mathrm{j}=0}^{\mathrm{t}-\mathrm{s}-1} \sum_{\mathrm{m}=0}^{\mathrm{n}-\mathrm{t}} \sum_{\mathrm{L}=0}^{\mathrm{k}_{3}} \sum_{\mathrm{h}=0}^{\alpha(\mathrm{t}-\mathrm{s}-\mathrm{j}+\mathrm{m})-1} \sum_{\mathrm{p}=0}^{\mathrm{k}_{2}} \sum_{\mathrm{q}=0}^{\alpha(\mathrm{s}-\mathrm{r}-\mathrm{i}+\mathrm{j})-1} \sum_{\mathrm{v}=0}^{\mathrm{k}_{1}} \sum_{\mathrm{w}=0}^{\alpha(\mathrm{i}+\mathrm{r})-1} \\
& \times(-1)^{\mathrm{i}+\mathrm{j}+\mathrm{m}+\mathrm{L}+\mathrm{h}+\mathrm{p}+\mathrm{q}+\mathrm{v}+\mathrm{w}}\left(\begin{array}{c}
\mathrm{s}-\mathrm{r}-1 \\
\mathrm{i}
\end{array}\right)\left(\begin{array}{c}
\mathrm{t}-\mathrm{s}-1 \\
\mathrm{j}
\end{array}\right)\left(\begin{array}{c}
\mathrm{n}-\mathrm{t} \\
\mathrm{m}
\end{array}\right)\left(\begin{array}{c}
\mathrm{k}_{3} \\
1
\end{array}\right)\left(\begin{array}{c}
\alpha(\mathrm{t}-\mathrm{s}-\mathrm{j}+\mathrm{m})-1 \\
\mathrm{~h}
\end{array}\right) \\
& \times\left(\begin{array}{c}
\mathrm{k}_{1} \\
\mathrm{p}
\end{array}\right)\left(\begin{array}{c}
\alpha(\mathrm{s}-\mathrm{r}-\mathrm{i}+\mathrm{j})-1 \\
\mathrm{q}
\end{array}\right)\left(\begin{array}{c}
\mathrm{k}_{1} \\
\mathrm{v}(\mathrm{i}+\mathrm{r})-1 \\
\mathrm{w}
\end{array}\right) \\
& \times\left[\begin{array}{c}
1 \\
\left(1-\mathrm{k}_{3}+\mathrm{h} \theta+\theta\right)\left(\mathrm{L}+\mathrm{p}-\mathrm{k}_{2}-\mathrm{k}_{3}+\mathrm{h} \theta+\mathrm{q} \theta+2 \theta\right)\left(\mathrm{L}+\mathrm{p}+\mathrm{v}-\mathrm{k}_{1}-\mathrm{k}_{2}-\mathrm{k}_{3}+\mathrm{h} \theta+\mathrm{q} \theta+\mathrm{w} \theta+3 \theta\right)
\end{array}\right.
\end{aligned}
$$


Proof: we know that

$$
\begin{aligned}
& \mu_{\mathrm{r}, \mathrm{s}, \mathrm{t}: \mathrm{n}}^{\left(\mathrm{k}_{1}, \mathrm{k}_{2}, \mathrm{k}_{3}\right)}=\int_{0}^{\infty} \int_{\mathrm{x} y}^{\infty} \int_{\mathrm{y}}^{\infty} \mathrm{x}^{\mathrm{k}_{1}} \mathrm{y}^{\mathrm{k}_{2}} \mathrm{z}^{\mathrm{k}_{3}} \mathrm{f}_{\mathrm{r}, \mathrm{s}, \mathrm{t:n}}(\mathrm{x}, \mathrm{y}, \mathrm{z}) \mathrm{dx} \mathrm{dy} \mathrm{dz} \\
& =\mathrm{C}_{\mathrm{r}, \mathrm{s}, \mathrm{t}: \mathrm{n}} \alpha^{3} \theta^{3} \lambda^{3} \sum_{\mathrm{i}=0}^{\mathrm{s}-\mathrm{r}-1} \sum_{\mathrm{j}=0}^{\mathrm{t}-\mathrm{s}-1} \sum_{\mathrm{m}=0}^{\mathrm{n}-\mathrm{t}}(-1)^{\mathrm{i}+\mathrm{j}+\mathrm{m}}\left(\begin{array}{c}
\mathrm{s}-\mathrm{r}-1 \\
\mathrm{i}
\end{array}\right)\left(\begin{array}{c}
\mathrm{t}-\mathrm{s}-1 \\
\mathrm{j}
\end{array}\right)\left(\begin{array}{c}
\mathrm{n}-\mathrm{t} \\
\mathrm{m}
\end{array}\right) \\
& \times \int_{0}^{\infty} \mathrm{x}^{\mathrm{k}_{1}}\left[1-(1+\lambda \mathrm{x})^{-\theta}\right]^{\alpha(\mathrm{i}+\mathrm{r})-1}[1+\lambda \mathrm{x}]^{-(\theta+1)} \mathrm{I}(\mathrm{x}) \mathrm{I}(\mathrm{y}) \mathrm{dx}
\end{aligned}
$$

Where $I(x)=\int_{x}^{\infty} y^{k_{2}}(1+\lambda y)^{-(\theta+1)}\left[1-(1+\lambda y)^{-\theta}\right]^{\alpha(s-r-i+j)-1} I(y) d y$ and $\quad I(y)=\int_{0}^{\infty} z^{k_{3}}(1+\lambda z)^{-(\theta+1)}\left[1-(1+\lambda z)^{-\theta}\right]^{\alpha(t-s-j+m)-1} d z$

In I (y), put $(1+\lambda z)^{-\theta}=\mathrm{v}_{1} \cdot(1+\lambda \mathrm{y})^{-\theta}<\mathrm{v}_{1}<0 ، \lambda \theta(1+\lambda \mathrm{z})^{-(\theta+1)} \mathrm{dz}=-\mathrm{dv}_{1}$ and $\mathrm{z}=\left(\mathrm{v}_{1}^{-\frac{1}{\theta}}-1\right) / \lambda$, substituting in $\mathrm{I}(\mathrm{y})$, using the binomial expansion and then simplifying the resulting, then

$$
\begin{aligned}
I(y)= & \lambda^{-\mathrm{k}_{3}-1} \sum_{\mathrm{L}=0}^{\mathrm{k}_{3}} \sum_{\mathrm{h}=0}^{\alpha(\mathrm{t}-\mathrm{s}-\mathrm{j}+\mathrm{m})-1}(-1)^{\mathrm{L}+\mathrm{h}}\left(\begin{array}{c}
\mathrm{k}_{3} \\
\mathrm{~L}
\end{array}\right)\left(\begin{array}{c}
\alpha(\mathrm{t}-\mathrm{s}-\mathrm{j}+\mathrm{m})-1 \\
\mathrm{~h}
\end{array}\right)\left[\frac{1}{\mathrm{~L}-\mathrm{k}_{3}+\mathrm{h} \theta+\theta}\right] \\
& \times\left[(1+\lambda \mathrm{y})^{-\theta}\right]^{\left(\frac{\mathrm{L}-\mathrm{k}_{3}+\mathrm{h} \theta+\theta}{\theta}\right)}
\end{aligned}
$$

Substituting I (y) in I (x), then

$$
\begin{aligned}
I(x)= & \lambda^{-k_{3}-1} \sum_{L=0}^{k_{3}} \sum_{h=0}^{\alpha(t-s-j+m)-1}(-1)^{L+h}\left(\begin{array}{c}
k_{3} \\
L
\end{array}\right)\left(\begin{array}{c}
\alpha(t-s-j+m)-1 \\
h
\end{array}\right)\left[\frac{1}{L-k_{3}+h \theta+\theta}\right] \\
& \times \int_{x}^{\infty} y^{k_{2}}(1+\lambda y)^{-(\theta+1)}[1-(1+\lambda y)]^{\alpha(s-r-i+j)-1}\left[(1+\lambda y)^{-\theta}\right]^{\left(\frac{L_{-}-k_{3}+h \theta+\theta}{\theta}\right)} d y
\end{aligned}
$$

In I $(x)$, put $(1+\lambda y)^{-\theta}=\mathrm{v}_{2} \cdot(1+\lambda y)^{-\theta}<\mathrm{v}_{2}<0 ، \lambda \theta(1+\lambda \mathrm{y})^{-(\theta+1)} \mathrm{dy}=-\mathrm{dv}_{2}$ and

$$
\begin{aligned}
& \mathrm{y}=\left(\mathrm{v}_{2}{ }^{-\frac{1}{\theta}}-1\right) / \lambda \text {, then } \\
& I(x)=\frac{\lambda^{-\left(k_{2}+k_{3}\right)}}{\lambda^{2}} \sum_{L=0}^{k_{3}} \sum_{h=0}^{\alpha(t-s-j+m)-1} \sum_{p=0}^{k_{2}} \sum_{q=0}^{\alpha(s-r-i+j)-1}(-1)^{L+h+p+q}\left(\begin{array}{c}
k_{3} \\
L
\end{array}\right)\left(\begin{array}{c}
\alpha(t-s-j+m)-1 \\
h
\end{array}\right) \\
& \times\left(\begin{array}{c}
\mathrm{k}_{2} \\
\mathrm{p}
\end{array}\right)\left(\begin{array}{c}
\alpha(\mathrm{s}-\mathrm{r}-\mathrm{i}+\mathrm{j})-1 \\
\mathrm{q}
\end{array}\right)\left[\frac{1}{\left(\mathrm{~L}-\mathrm{k}_{3}+\mathrm{h} \theta+\theta\right)\left(\mathrm{L}+\mathrm{p}-\mathrm{k}_{2}-\mathrm{k}_{3}+\mathrm{h} \theta+\mathrm{q} \theta+2 \theta\right)}\right] \\
& \times\left[(1+\lambda \mathrm{y})^{-\theta}\right]^{\left(\frac{\mathrm{L}+\mathrm{p}-\mathrm{k}_{2}-\mathrm{k}_{3}+\mathrm{h} \theta+\mathrm{q} \theta+2 \theta}{\theta}\right)}
\end{aligned}
$$


Substituting I (x) in (18-A), we found that

$$
\begin{aligned}
& \mu_{\mathrm{r}, \mathrm{s}, \mathrm{t}: \mathrm{n}}^{\left(\mathrm{k}_{1}, \mathrm{k}_{2}, \mathrm{k}_{3}\right)}=\mathrm{C}_{\mathrm{r}, \mathrm{s}, \mathrm{t}: \mathrm{n}} \alpha^{3} \theta^{3} \lambda \lambda^{-\left(\mathrm{k}_{2}+\mathrm{k}_{3}\right)} \sum_{\mathrm{i}=0}^{\mathrm{s}-\mathrm{r}-1} \sum_{\mathrm{j}=0}^{\mathrm{t}-\mathrm{s}-1} \sum_{\mathrm{m}=0}^{\mathrm{n}-\mathrm{t}} \sum_{\mathrm{L}=0}^{\mathrm{k}_{3}} \sum_{\mathrm{h}=0}^{\alpha(\mathrm{t}-\mathrm{s}-\mathrm{j}+\mathrm{m})-1} \sum_{\mathrm{p}=0}^{\mathrm{k}_{2}} \\
& \times \sum_{\mathrm{q}=0}^{\alpha(\mathrm{s}-\mathrm{r}-\mathrm{i}+\mathrm{j})-1}\left(\begin{array}{c}
\mathrm{s}-\mathrm{r}-1 \\
\mathrm{i}
\end{array}\right)\left(\begin{array}{c}
\mathrm{t}-\mathrm{s}-1 \\
\mathrm{j}
\end{array}\right)\left(\begin{array}{c}
\mathrm{n}-\mathrm{t} \\
\mathrm{m}
\end{array}\right)\left(\begin{array}{c}
\mathrm{k}_{3} \\
\mathrm{~L}
\end{array}\right)\left(\begin{array}{c}
\alpha(\mathrm{t}-\mathrm{s}-\mathrm{j}+\mathrm{m})-1 \\
\mathrm{~h}
\end{array}\right)\left(\begin{array}{c}
\mathrm{k}_{2} \\
\mathrm{p}
\end{array}\right) \\
& \times\left(\begin{array}{c}
\alpha(\mathrm{t}-\mathrm{s}-\mathrm{i}+\mathrm{j})-1 \\
\mathrm{q}
\end{array}\right)(-1)^{\mathrm{i}+\mathrm{j}+\mathrm{m}+\mathrm{l}+\mathrm{h}+\mathrm{p}+\mathrm{q}}\left[\frac{1}{\left(\mathrm{~L}-\mathrm{k}_{3}+\mathrm{h} \theta+\theta\right)\left(\mathrm{L}+\mathrm{p}-\mathrm{k}_{2}-\mathrm{k}_{3}+\mathrm{h} \theta+\mathrm{q} \theta+2 \theta\right)}\right] \\
& \times \int_{0}^{\infty} \mathrm{x}^{\mathrm{k}_{1}}(1+\lambda \mathrm{x})^{-(\theta+1)}\left[1-(1+\lambda \mathrm{x})^{-\theta}\right]^{\alpha(\mathrm{i}+\mathrm{r})-1}\left[(1+\lambda \mathrm{x})^{-\theta}\right]^{\left(\mathrm{L}+\mathrm{p}-\mathrm{k}_{2}-\mathrm{k}_{3}+\mathrm{h} \theta+\mathrm{q} \theta+2 \theta\right) / \theta} \mathrm{dx}
\end{aligned}
$$

By using the same way that we used to get I (y) and I (x), we obtain (18).

\section{Recurrence relations for single and product moments}

In this section, the recurrence relations for the single and product moment of the ELD are established as follows: from (1) and (2), we have when $\theta$ is a positive integer

$$
\begin{aligned}
& F(x)=\frac{1}{\alpha \theta \lambda}\left[1-(1+\lambda x)^{-\theta}\right][1+\lambda x]^{\theta+1} f(x) \\
& =\frac{1}{\alpha \theta \lambda}\left[(1+\lambda x)^{\theta+1}-(1+\lambda x)\right] \mathrm{f}(\mathrm{x}) \\
& =\frac{1}{\alpha \theta \lambda}\left[\sum_{i=0}^{\theta+1}\left(\begin{array}{c}
\theta+1 \\
i
\end{array}\right)(\lambda x)^{i}-(1+\lambda x)\right] f(x) \\
& F(x)=\frac{1}{\alpha}\left[x+\frac{1}{\theta \lambda} \sum_{i=2}^{\theta+1}\left(\begin{array}{c}
\theta+1 \\
i
\end{array}\right)(\lambda x)^{i}\right] f(x)
\end{aligned}
$$

Theorem 4.1. for ELD and for $2 \leq \mathrm{r} \leq \mathrm{n}, \theta$ is a positive integer

$$
\mu_{r: n}^{(\mathrm{k})}=\frac{\mathrm{k}}{\alpha \theta(\mathrm{r}-1)} \sum_{\mathrm{i}=2}^{\theta+1}\left(\begin{array}{c}
\theta+1 \\
\mathrm{i}
\end{array}\right) \lambda^{\mathrm{i}-1} \mu_{\mathrm{r}-1: \mathrm{n}}^{(\mathrm{k}+\mathrm{i}-1)}+\left(\frac{\mathrm{k}}{\alpha(\mathrm{r}-1)}+1\right) \stackrel{\mathrm{r}-1: \mathrm{n}}{\mathrm{k})}^{\mathrm{k}}
$$

Proof

$\underset{r-1: n}{(k)}=\frac{n !}{(r-2) !(n-r+1) !} \int_{0}^{\infty} x^{k}[F(x)]^{r-2}[1-F(x)]^{n-r+1} f(x) d x$

Integration by parts treating $[F(x)]^{r-2} f(x) d x$ for integration and the rest of the integrand for differentiation, we get

$$
\begin{aligned}
\mu_{r-1: n}^{(k)}= & \frac{n !}{(r-2) !(n-r+1) !}\left\{\frac{n-r+1}{r-1} \int_{0}^{\infty} x^{k}[F(x)]^{r-1}[1-F(x)]^{n-r} f(x) d x-\frac{k}{r-1} \int_{0}^{\infty} x^{k-1}\right. \\
& {\left.[F(x)]^{r-2} F(x) \times[1-F(x)]^{n-r+1} d x\right\} }
\end{aligned}
$$


By using (19), we obtain

$$
\begin{aligned}
\mu_{\mathrm{r}-1: \mathrm{n}}^{(\mathrm{k})}= & \frac{\mathrm{n} !}{(\mathrm{r}-1) !(\mathrm{n}-\mathrm{r}) !} \int_{0}^{\infty} \mathrm{x}^{\mathrm{k}}[\mathrm{F}(\mathrm{x})]^{\mathrm{r}-1}[1-\mathrm{F}(\mathrm{x})]^{\mathrm{n}-\mathrm{r}} \mathrm{f}(\mathrm{x}) \mathrm{dx}-\frac{\mathrm{n} !}{(\mathrm{r}-2) !(\mathrm{n}-\mathrm{r}+1) !} \cdot \frac{\mathrm{k}}{(\mathrm{r}-1)} \\
& \times \int_{0}^{\infty} \mathrm{x}^{\mathrm{k}-1}[\mathrm{~F}(\mathrm{x})]^{\mathrm{r}-2}\left[\frac{\mathrm{x}}{\alpha}+\frac{1}{\alpha \lambda \theta} \sum_{\mathrm{i}=2}^{\theta+1}\left(\begin{array}{c}
\theta+1 \\
\mathrm{i}
\end{array}\right)(\lambda \mathrm{x})^{\mathrm{i}}\right] \mathrm{f}(\mathrm{x})[1-\mathrm{F}(\mathrm{x})]^{\mathrm{n}-\mathrm{r}+1} \mathrm{dx} \\
\mu_{\mathrm{r}-1: \mathrm{n}}^{(\mathrm{k})} & =\mu_{\mathrm{r}: \mathrm{n}}^{(\mathrm{k})}-\frac{\mathrm{k}}{\alpha(\mathrm{r}-1)} \mu_{\mathrm{r}-1: \mathrm{n}}^{(\mathrm{k})}-\frac{\mathrm{k}}{\alpha \theta(\mathrm{r}-1)} \sum_{\mathrm{i}=2}^{\theta+1}\left(\begin{array}{c}
\theta+1 \\
\mathrm{i}
\end{array}\right) \lambda^{\mathrm{i}-1} \mu_{\mathrm{r}-1: \mathrm{n}}^{(\mathrm{k}+\mathrm{i}-1)} \\
\text { Then } & \\
\mu_{\mathrm{r}: \mathrm{n}}^{(\mathrm{k})}= & \frac{\mathrm{k}}{\alpha \theta(\mathrm{r}-1)} \sum_{\mathrm{i}=2}^{\theta+1}\left(\begin{array}{c}
\theta+1 \\
\mathrm{i}
\end{array}\right) \lambda^{\mathrm{i}-1} \mu_{\mathrm{r}-1: \mathrm{n}}^{(\mathrm{k}+\mathrm{i}-1)}+\left(\frac{\mathrm{k}}{\alpha(\mathrm{r}-1)}+1\right) \mu_{\mathrm{r}-1: \mathrm{n}}^{(\mathrm{k})}
\end{aligned}
$$

Also, by using the same way, we get

$$
\mu_{\mathrm{n}-\mathrm{r}+2: \mathrm{n}}^{(\mathrm{k})}=\frac{\mathrm{k}}{\alpha \theta(\mathrm{n}-\mathrm{r}+1)} \sum_{\mathrm{i}=2}^{\theta+1}\left(\begin{array}{c}
\theta+1 \\
\mathrm{i}
\end{array}\right) \lambda^{\mathrm{i}-1} \stackrel{\mathrm{k}}{\mu}_{\mathrm{n}-\mathrm{r}+1}^{\mathrm{k}+\mathrm{i}-1}+\left(\frac{\mathrm{k}}{\alpha(\mathrm{n}-\mathrm{r}+1)}+1\right) \stackrel{(\mathrm{k})}{\mu_{\mathrm{n}-\mathrm{r}+\mathrm{l}: \mathrm{n}}}
$$

Note that, when $\alpha=1$ in (20), we get the recurrence relations for single moments from lomax distribution and also, when $\lambda=1$ in (20) we get the recurrence relations for single moments from the exponentiated pareto distribution.

Theorem 4.2. For the distribution as given (1) and for $1 \leq \mathrm{r}<\mathrm{s} \leq \mathrm{n}$ and $\theta$ is a positive integer

$$
\mu_{\mathrm{r}+1, \mathrm{~s}: \mathrm{n}}^{\left(\mathrm{k}_{1}, \mathrm{k}_{2}\right)}=\frac{\mathrm{k}_{1}}{\alpha \theta \mathrm{r}} \sum_{\mathrm{i}=2}^{\theta+1}\left(\begin{array}{c}
\theta+1 \\
\mathrm{i}
\end{array}\right) \lambda^{\mathrm{i}-1} \mu_{\mathrm{r}, \mathrm{s}: \mathrm{n}}^{\left(\mathrm{k}_{1}+\mathrm{i}-1, \mathrm{k}_{2}\right)}+\left(\frac{\mathrm{k}}{\alpha \mathrm{r}}+1\right) \mu_{\mathrm{r}, \mathrm{s}: \mathrm{n}}^{\left(\mathrm{k}_{1}, \mathrm{k}_{2}\right)}
$$

Proof:

$\mu_{\mathrm{r}, \mathrm{s}: \mathrm{n}}^{\left(\mathrm{k}_{1}, \mathrm{k}_{2}\right)}=\mathrm{C}_{\mathrm{r}, \mathrm{s}: \mathrm{n}} \int_{0}^{\infty} \mathrm{y}^{\mathrm{k}_{2}}[1-\mathrm{F}(\mathrm{y})]^{\mathrm{n}-\mathrm{s}} \mathrm{f}(\mathrm{y}) \mathrm{I}(\mathrm{y}) \mathrm{dy}$

Where $I(y)=\int_{0}^{y} x^{k_{1}}[F(y)-F(x)]^{s-r-1}[F(x)]^{r-1} f(x) d x$

Solving the integral in I (y) by parts, we get

$$
\begin{aligned}
& I(y)=\frac{s-r-1}{r} \int_{0}^{y} x^{k_{1}}[F(x)]^{r}[F(y)-F(x)]^{s-r-2} f(x) d x \\
& -\frac{k_{1}}{r} \int_{0}^{y} x^{k_{1}-1}[F(y)-F(x)]^{s-r-1}[F(x)]^{r-1} F(x) d x
\end{aligned}
$$

on substituting for $\mathrm{F}(\mathrm{x})$ from (19) in $\mathrm{I}(\mathrm{y})$ and substitute it in (21-A), we get

$$
\begin{aligned}
& \mu_{r, s: n}^{\left(k_{1}, k_{2}\right)}=\frac{n !}{r !(s-r-2) !(n-s) !} \int_{0}^{\infty} \int_{0}^{y} x^{k_{1}} y^{k_{2}}[F(x)]^{r}[F(y)-F(x)]^{s-r-2}[1-F(y)]^{n-s} f(x) f(y) d x d y \\
& \quad-\frac{k_{1}}{\alpha r} C_{r, s: n} \int_{00}^{\infty y} x^{k_{1}} y^{k_{2}}[F(x)]^{r-1}[F(y)-F(x)]^{s-r-1}[1-F(y)]^{n-s} f(x) f(y) d x d y \\
& -\frac{k_{1}}{\alpha \theta r} \sum_{i=2}^{\theta+1}\left(\begin{array}{c}
\theta+1 \\
i
\end{array}\right) \lambda^{i-1} \int_{0}^{\infty} \int_{0}^{\infty y} x^{k_{1}+i-1} y^{k_{2}}[F(x)]^{r-1}[F(y)-F(x)]^{s-r-1}[1-F(y)]^{n-s} f(x) f(y) d x d y
\end{aligned}
$$

Then 
$\mu_{\mathrm{r}+1, \mathrm{~s} n}^{\left(\mathrm{k}_{1}, \mathrm{k}_{2}\right)}=\frac{\mathrm{k}_{1}}{\alpha \theta \mathrm{r}} \sum_{\mathrm{i}=2}^{\theta+1}\left(\begin{array}{c}\theta+1 \\ \mathrm{i}\end{array}\right) \lambda^{\mathrm{i}-1} \mu_{\mathrm{r}, \mathrm{s}: \mathrm{n}}^{\left(\mathrm{k}_{1}+\mathrm{i}-1, \mathrm{k}_{2}\right)}+\left(\frac{\mathrm{k}_{1}}{\alpha \mathrm{r}}+1\right) \mu_{\mathrm{r}, \mathrm{s}: \mathrm{n}}^{\left(\mathrm{k}_{1}, \mathrm{k}_{2}\right)}$

Note that, when $\alpha=1, \alpha=\lambda=1$ and $\lambda=1$, we obtain the recurrence relations for product moments from lomax distribution, standard pareto distribution and exponentiated pareto distribution respectively.

\section{Percentage Points of order statistics}

The cumulative distribution function of $\mathrm{x}_{\mathrm{r}: \mathrm{n}}, 1 \leq \mathrm{r} \leq \mathrm{n}$ is given by

$$
\mathrm{F}_{\mathrm{r}: \mathrm{n}}(\mathrm{x})=\mathrm{I}_{\mathrm{F}(\mathrm{x})}(\mathrm{r}, \mathrm{n}-\mathrm{r}+1)
$$

Where $\mathrm{F}(\mathrm{x})=\mathrm{F}(\mathrm{x} ; \alpha, \lambda, \theta)$ is cdf given in $(2)$ and

$$
I_{F(x)}(r, n-r+1)=[B(r, n-r+1)] \int_{0}^{-1} t^{\mathrm{F}(\mathrm{x})}(1-t)^{(\mathrm{n}-\mathrm{r}+1)-1} \mathrm{dt}
$$

Where $I_{F(x)}(r, n-r+1)$ is the incomplete beta function. Therefore, the $100 p^{\text {th }}$ percentile of $x_{r: n}$ for given $n, r$ and $p$ can be obtained by solving the following equation (Raqab 1998).

$$
\mathrm{I}_{\mathrm{F}(\mathrm{x})}(\mathrm{r}, \mathrm{n}-\mathrm{r}+1)=\mathrm{p}
$$

The percentage points can be calculated from (23) either by using the tables of incomplete beta function prepared by Pearson (1934) or by using the algorithm given by cran et al (1977). However for $r=1$, equation (23) reduces to $\left.1-p=\left[1-(1+\lambda x)^{-\theta}\right)^{\alpha}\right]^{n}$. Thus, the percentage point of the smallest order statistics $\mathrm{x}_{1: \mathrm{n}}$ is given by

$$
\mathrm{x}_{1: \mathrm{n}, \mathrm{p}}=\frac{\left[1-\left(1-(1-\mathrm{p})^{1 / \mathrm{n}}\right)^{1 / \alpha}\right]^{-1 / \theta}-1}{\lambda}
$$

and let $r=n$, equation (23) reduces to $P=[F(x)]^{n}=\left[1-(1+\lambda x)^{-\theta}\right]^{\alpha n}$. Thus, the percentage point of the largest order statistic $x_{n: n}$ is given by

$$
x_{n: n, p}=\frac{\left(1-p^{1 / \alpha n}\right)^{-1 / \theta}-1}{\lambda}
$$

\begin{tabular}{|c|c|c|c|c|c|c|c|c|c|}
\hline \multirow{2}{*}{$\mathbf{n}$} & \multicolumn{9}{|c|}{$\mathbf{X}_{1: \mathbf{n}, \mathbf{p}}$} \\
\hline & $P=0.1$ & $P=0.2$ & $P=0.3$ & $\mathrm{P}=0.4$ & $P=0.5$ & $P=0.6$ & $P=0.7$ & $P=0.8$ & $P=0.9$ \\
\hline 2 & 0.229 & 0.347 & 0.463 & 0.588 & 0.733 & 0.912 & 1.152 & 1.517 & 2.242 \\
\hline 5 & 0.144 & 0.210 & 0.269 & $\mathbf{0 . 3 3 0}$ & 0.395 & 0.471 & 0.565 & 0.694 & 0.916 \\
\hline 10 & 0.104 & 0.148 & 0.187 & 0.225 & 0.265 & 0.310 & 0.363 & 0.434 & 0.548 \\
\hline 15 & 0.086 & 0.122 & 0.153 & 0.183 & 0.213 & 0.247 & 0.287 & 0.339 & 0.421 \\
\hline \multicolumn{10}{|c|}{$\mathbf{X}_{\mathbf{n}: \mathbf{n}, \mathbf{p}}$} \\
\hline 2 & 0.786 & 1.136 & 1.496 & 1.91 & 2.423 & 3.111 & 4.127 & 5.889 & 10.167 \\
\hline 5 & 1.901 & 2.577 & 3.261 & 4.041 & 5.004 & 6.287 & 8.176 & 11.44 & 19.344 \\
\hline 10 & 3.379 & 4.467 & 5.546 & 6.812 & 8.348 & 10.392 & 13.401 & 18.591 & 31.151 \\
\hline 15 & 4.631 & 6.062 & 7.505 & 9.144 & 11.16 & 13.84 & 17.79 & 24.60 & 41.06 \\
\hline
\end{tabular}

The following tables (2) and (3) gives values of $100 \mathrm{p}^{\text {th }}$ percentage points of $\mathrm{X}_{1: \mathrm{n}, \mathrm{p}}$ and $\mathrm{X}_{\mathrm{n}: \mathrm{n}, \mathrm{p}}$ for $\mathrm{n}=2,5,10,15$ and for $\mathrm{P}=0.1$ to 0.9 when $\theta=1.5,5, \lambda=1.2,2$ and $\alpha=2,2.5$

Table (2): $100 p^{\text {th }}$ percentage points of $X_{1: n, p}$ and $X_{n: n, p}$ for $\lambda=1.2, \alpha=2.5$ and $\theta=1.5$ 
Table (3): $100 p^{\text {th }}$ percentage points of $X_{1: n, p}$ and $X_{n: n, p}$ for $\lambda=2, \alpha=2$ and $\theta=5$

\begin{tabular}{|c|c|c|c|c|c|c|c|c|c|}
\hline \multirow{2}{*}{$\mathbf{n}$} & \multicolumn{9}{|c|}{$\mathbf{X}_{1: \mathrm{n}, \mathrm{p}}$} \\
\hline & $P=0.1$ & $P=0.2$ & $P=0.3$ & $\mathrm{P}=0.4$ & $P=0.5$ & $P=0.6$ & $P=0.7$ & $\mathrm{P}=0.8$ & $P=0.9$ \\
\hline 2 & 0.026 & 0.041 & 0.055 & 0.069 & 0.084 & 0.102 & 0.125 & 0.156 & 0.210 \\
\hline 5 & 0.016 & 0.024 & 0.031 & 0.039 & 0.047 & 0.055 & 0.066 & 0.08 & 0.103 \\
\hline 10 & 0.011 & 0.016 & 0.021 & 0.026 & 0.031 & 0.036 & 0.043 & 0.051 & 0.064 \\
\hline 15 & 0.009 & 0.013 & 0.017 & 0.021 & 0.024 & 0.029 & 0.034 & 0.04 & 0.05 \\
\hline \multicolumn{10}{|c|}{$\mathbf{X}_{\mathrm{n}: \mathrm{n}, \mathrm{p}}$} \\
\hline 2 & 0.09 & 0.124 & 0.155 & 0.187 & 0.222 & 0.264 & 0.318 & 0.396 & 0.537 \\
\hline 5 & 0.186 & 0.232 & 0.273 & 0.314 & 0.359 & 0.411 & 0.477 & 0.572 & 0.744 \\
\hline 10 & 0.279 & 0.334 & 0.382 & 0.431 & 0.483 & 0.544 & 0.621 & 0.73 & 0.928 \\
\hline 15 & 0.342 & 0.402 & 0.455 & 0.508 & 0.565 & 0.631 & 0.715 & 0.834 & 1.049 \\
\hline
\end{tabular}

When $1<\mathrm{r}<\mathrm{n}$, the percentage points can also be obtained by using t-approximation to in complete beta function (Ojo 1988) as follows: Let $\mathrm{T}_{\mathrm{v}}$ be a $t$ random variable with $\mathrm{v}$ degrees of freedom which is obtained by equatating the coefficient of kurtosis of the generalized logistic to that of the t-distribution. Denote the $\mathrm{r}^{\text {th }}$ cumulant of the logistic distribution by $\mathrm{k}_{\mathrm{r}}$. The approximate expression for the $\mathrm{F}_{\mathrm{r}: \mathrm{n}}(\mathrm{x})$ is found to be

Where

$$
\begin{aligned}
& F_{r: n}(x)=p\left[T_{v} \leq C\left(\ln \frac{F(x)}{1-F(x)}-k_{1}\right)\right] \\
& C=\sqrt{\frac{v}{k_{2}(v-2)}}
\end{aligned}
$$

This immediately given

$$
\mathrm{x}_{\mathrm{r}: \mathrm{n}, \mathrm{p}}=\frac{\left[1-\left(1+\exp \left[-\left(\frac{\mathrm{T}_{\mathrm{v}}}{\mathrm{C}}+\mathrm{k}_{1}\right)\right]\right)^{-1 / \alpha}\right]^{-1 / \theta}-1}{\lambda}
$$

Approximation to percentiles of order statistics $x_{r: n},(1 \leq r \leq n)$ can be obtained from (24) by using t-table.

\section{Conclusion}

This study deals with the order statistics from the exponentiated Lomax distribution (ELD). Explicit forms for the single, product and triple moments of the order statistics from ELD are derived. Coefficients of skewness and kurtosis are calculated for different values of sample size and distribution parameters. Some recurrence relations for both single and product moments are established. Also, the percentage points of the $\mathrm{r}^{\text {th }}$ order statistic from ELD are presented and computed for the smallest and the largest order statistics at different values of sample size and distribution parameters.

\section{References}

[1] Abdul-Moniem, I.B. and Abdel-Hameed, H.F.,(2012) "on exponentiated Lomax distribution", International Journal of mathematical archive, 3, 2144-2150.

[2] Al-Zahrani, B., Dar, J.G and Al-Sobhi, M.,(2015) "Moment of order statistics of the poisson-Lomax distribution", advances in mathematics scientific, 4, 49-55. 
[3] Arnold, B.C., Balakirshnan, N. and Nagraja, N.H., (1992), A first course in order statistics, John Wiley and Sons, New York.

[4] Athar, H., and Nayabuddin,(2014) "a note on exact moments of order statistics from exponentiated log-logistic distribution", probstat forum, 7, 39- 44.

[5] - Balakirshnan, N., Malik, H.J and Ahmed, S.E.(1988), "recurrence relations identities for moments of order statistics specific continuous distributions", commun . statist . theo. Meth., 17, $2657-2694$.

[6] - Balakrishnan, N. and Cohen, A.C., (1991), order statistics and inference: estimation method, academic press, San Diego .

[7] Balakrishnan, N. and Chan, P.S, (1998), "Log-gamma order statistics and linear estimation of parameters", In: Balakrishnan, N. and Rao, C.R. (eds), handbook of statistics, Elsevier science, Amsterdam, 17, 61-83.

[8] Cran, G.W. Martin, K.J. and Thomas, G.E., (1977), "A remark on algorithms", Algorithm As 109, Appl.statist. , 26, 111-114

[9] David, A. and Nagaraja, H.N., (2003), order statistics, $3^{\text {rd }}$ edition, Wiley, New Jersey.

[10] Joshi, P.C. and Balakrishnan, N.,(1982), "recurrence relations and identities for the product moments of order statistics", Sankhya B, 44, 39-49.

[11] Khan, R.W.,Anwar, Z. and Athar,H., (2008), "recurrence relations for single and product moments of dual generalized order statistics from exponentiated weibull distribution", aligarh journal of statistics, 28, 37-45.

[12] Malik, H.J., (1966), "exact moments of order statistics from pareto distribution", Skand. Aktuarie Tidskr, 49, $144-157$.

[13] Ojo,M.O.,(1988), "a new approximation for the percentiles of the t-distribution", Technometrics, 28, $103-106$.

[14] Pearson, K.., (1934), Tables of the incomplete beta function, Cambridge University press, Cambridge .

[15] Raqab, M.Z., (1988), "order statistics from the Burr type X model", computers Math. Applic., 36, 111-120.

[16] Shawky, A.I. and Bakoban, R.A. (2009), "order statistics from exponentiated gamma distribution and associated inference", int. J. Contemp. Math. Sciences, 4, 71-91.

[17] Sultan, K.S. (2007) "order statistics from the generalized exponential distribution and applications", Communication in statistics - Theory and Methods, 36, 1409-1418.

[18] Tahir, M.H., Cordeiro, G.M Mansoor, M. and Zubair, M., (2015) "The weibull - Lomax distribution: properties and applications", Hacettepe Journal of mathematics and statistics, 44, 455-474. 